\title{
Occurrence of Haemophilus influenzae strains in three Brazilian states since the introduction of a conjugate Haemophilus influenzae type $b$ vaccine
}

A.E.C.C. de Almeida ${ }^{1}$, I. de Filippis ${ }^{1}$, A.O. de Abreu ${ }^{1}$,

D.G. Ferreira' ${ }^{1}$, A.L. Gemal ${ }^{1}$ and K.B.F. Marzochi ${ }^{2}$

\author{
${ }^{1}$ Instituto Nacional de Controle da Qualidade em Saúde, \\ Departamento de Microbiologia, ${ }^{2}$ Instituto de Pesquisas Evandro Chagas, \\ Fundação Oswaldo Cruz, Rio de Janeiro, RJ, Brasil
}

Correspondence

A.E.C.C. de Almeida

Av. Brasil, 4365, Manguinhos

21045-900 Rio de Janeiro, RJ

Brasil

Fax: +55-21-2290-0915

E-mail: eugenio@incqs.fiocruz.br

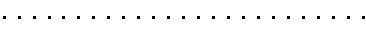

Received September 28, 2004 Accepted February 16, 2005

\section{Abstract}

Few vaccines in history have induced such a dramatic decline in incidence over such a short period of time as the Haemophilus influenzae type $\mathrm{b}$ (Hib) conjugate. This vaccine was introduced in 1988 in the United States, but only in 1999 was Hib immunization introduced by the Brazilian Ministry of Health as part of the routine infant National Immunization Program. The authors analyzed $229 \mathrm{H}$. influenzae (Hi) isolates from Public Health Laboratories in three Brazilian states: Pernambuco (Northeast, $N=54$ ), Santa Catarina (South, $N=19$ ), and Rio de Janeiro (Southeast, $N=156$ ). The isolates were collected from Brazilian children 0-10 years of age with meningitis and other infections from 1990 to 2003 and were part of the research collection of the National Institute of Quality Control in Health, FIOCRUZ. Bacterial strains were characterized by serotyping and biotyping. During the pre-vaccination period the prevalence infection due to Hib was of 165 isolates and only 2 non-b Hi among all the notified meningitis infections caused by Hi. Our results showed a significant decrease in the prevalence of Hib meningitis from 165 to 33 isolates after 1999. However, during the post-vaccination period of 2001-2003 we observed an increase in the number of non-b Hi isolates: only 2 non-b strains isolated from 1990 to 1999 and 29 from 1999 to 2003. Based on the present data, the authors emphasize the need for more sensitive epidemiological and bacteriological studies aiming the improvement of the available Hib vaccine, in order to protect the susceptible population to infections due to other serological types of $\mathrm{Hi}$ and the reevaluation of immunization schedules used by the National Immunization Program.

In Brazil, during the pre-vaccination period from 1990 to 1999, the coefficient of incidence (annual mean) of Haemophilus influenzae type $\mathrm{b}(\mathrm{Hib})$ meningitis in children up to 1 year and up to 4 years of age was
Key words

- Haemophilus influenzae

- Haemophilus influenzae non-b

- Hib conjugate vaccine

- Immunization

- Hib meningitis
22.3 and 8.8 cases per 100,000 inhabitants, respectively (CENEPI, FUNASA, Brazilian Ministry of Health). The lethality rates in these age groups were 19.9 and $17.1 \%$, respectively. In August 1999, the Hib conju- 
gate vaccine was introduced by the Brazilian Ministry of Health as part of the routine infant National Immunization Program. Children aged $<1$ year were scheduled to receive 3 vaccine doses given at 2-month intervals. The vaccination schedule followed in Brazil does not include the booster dose after 12 months. Children aged 12-23 months were scheduled to receive a single vaccine dose. Data from the Brazilian National Health Foundation/National Immunization Program (FUNASA/PNI/Ministry of Health) show that in 2002 and 2003, 92.91 and $95.99 \%$ of eligible children received the vaccine, respectively (1). Before the advent of Hib vaccination, pediatric invasive $H$. influenzae (Hi) disease was caused almost exclusively by Hib isolates. After the implementation of vaccination, serotype b disease decreased, and it has been predicted that after the eradication of Hib, other Haemophilus serotypes and non-typeable strains could become relatively more important $(2,3)$.

The current study was based on a collection of 229 Hi strains isolated from Brazilian children from 1990 to 2003 . We analyzed $\mathrm{Hi}$ strains (198 serotype b and 31 non-b strains) isolated from pediatric patients with meningitis $(\mathrm{N}=189)$, septicemia $(\mathrm{N}=3)$ and bronchial secretion $(\mathrm{N}=37)$. Clinical isolates were part of the research collection of the National Institute for Quality Control in Health (INCQS) at the Oswaldo Cruz Foundation (FIOCRUZ), Rio de Janeiro, RJ, Brazil. The Institute has monitored the evolution of phenotypic and genotypic characteristics of Hi strains before and after the introduction of the Hib vaccine by the National Immunization Program. The Institute receives clinical isolates from different regions of Brazil, mainly from the Regional Public Health Laboratories of Pernambuco (Northeast, $N=54$ ) and Santa Catarina (South, N = 19) and from two other Public Health Laboratories in Rio de Janeiro (Southeast, $\mathrm{N}=$ 156), Fernandes Figueira Institute, FIOCRUZ $(\mathrm{N}=46)$ and São Sebastião State Institute of
Infectology, State Secretary of Health (N = 110). Reference strains of Hi serotypes a to $f$ were obtained from the Reference Culture Collection of the INCQS/FIOCRUZ. All reference strains were originally from the American Type Culture Collection (USA).

$H$. influenzae isolates were confirmed by Gram staining, recognition of typical morphology after growing for $24 \mathrm{~h}$ in $5 \% \mathrm{CO}_{2}$ on chocolate agar consisting of Mueller Hinton agar base (Difco Laboratories, Detroit, MI, USA) enriched with $10 \%$ defibrinated horse blood and $1 \%$ IsoVitaleX. The biotyping analysis scheme used was based on the production of indole, ornithine decarboxylase, and urease. Serotypes of Hi strains were identified by reaction with monovalent antisera (Difco) against the capsular antigens a, b, c, d, e, and f. Non-typeable Hi strains were determined by lack of agglutination against any of the above-mentioned antisera $(4,5)$. Strains were stored at $-70^{\circ} \mathrm{C}$ in supplemented brain heart infusion broth containing $20 \%$ glycerol.

The frequency of serotypes and source of isolates are shown in Table 1 . We note that 165 of 167 strains maintained in our collection from the pre-vaccination period (19901999) are serotype b and only two are non-b strains (type a). These strains were isolated from children with meningitis aged one month to 10 years. Of these, $94 \%(\mathrm{~N}=157)$ were from children under 5 years of age following the introduction of $\mathrm{Hib}$ conjugate vaccine in Brazil (1999). Thus, our findings show an expected and rapid reduction in the number of invasive Hib infections (from 165 to 33), but other $\mathrm{Hi}$ serotypes and non-typeable strains showed increased isolation rates compared to the pre-vaccination period (Table 1). Based on data from the Brazilian National Health Foundation (FUNASA/Ministry of Health), following the introduction of $\mathrm{Hib}$ conjugate vaccine, the incidence of $\mathrm{Hib}$ meningitis in Brazil decreased by $83 \%$ in 2 years, from 1368 cases in 1999 to 234 cases in 2001 (1). 
During the period from 2001 to 2003 , we observed the appearance of other serotypes (non-b Hi) as well as an upward trend in both type $b$ and non-typeable Hi. In Brazil no epidemiological pattern has been determined for the country as a whole, suggesting differences in the quality of meningitis surveillance programs in each Brazilian geographic area (6). The Hib strains causing invasive disease during this period may be representative of the pool of strains circulating among carriers, or they may have been a subset of these carrier strains. It is also possible that the invasive strains were introduced from an unrecognized source. It is therefore of interest to determine differences between invasive and carrier Hib strains. Invasive Hi disease is now associated primarily with nontypeable Hi or with Hi of capsule types a, c, $\mathrm{d}$, e, and especially $\mathrm{f}$ (4). An adequate epidemiological surveillance system would be helpful to evaluate the role of these non-b Hi and non-typeable Hi strains as significant pathogens showing an increase in isolation rates.

It is important to determine the potential pathogenicity of Hi strains by encouraging the use of molecular typing methods for molecular epidemiology studies based on geographic origin and isolation periods. It is also important to investigate associations between the types/biotypes and clinical manifestations and age groups during the postvaccination versus the pre-vaccination period. We call attention to the fact that severe forms of disease are still present during the post-vaccination period. Table 1 shows that, despite the drastic decrease of reported cases between the pre-vaccination (1990-1999) and post-vaccination (2001-2003) periods, 22 out of 25 strains isolated from meningitis and septicemia were type b, 1 was type a, 1 type $\mathrm{c}$, and 1 type $\mathrm{d}$. There are few publications about Brazilian Hib strains, and one of the latest studies examining strains isolated in the Northeast region of Brazil showed an increase in type a isolates after vaccination against $\operatorname{Hib}(7,8)$.

Recent studies show an increase of nonb Hi isolates, suggesting possible serotype replacement, a potential concern for public health surveillance, since so far there are no vaccines available against serotypes a, c, d, $e$, and $f(4,5,9)$. Other studies show the reemergence of Hib strains among vaccinated patients in different countries, despite the different vaccination strategies used in their respective Immunization Programs $(10,11)$. Some reports have described increased rates of non-b Hi invasive disease in regions where Hib conjugate vaccines have been used $(12,13)$ and outbreaks of Hib disease have been reported (14). Results reported by the Centers for Disease Control show that because Hib vaccines protect against type $b$ and not against other Hi strains, serotyping of all $\mathrm{Hi}$ isolates from patients with invasive disease is necessary to monitor the effectiveness of the vaccination program and the national progress towards Hib elimination. Serotype information is needed to measure the sensitivity of the surveillance system and to detect the emergence of invasive disease from non-type b Hi strains (15).

Biotyping of Hi isolates can be used for epidemiological research purposes, but it is of minor value for patients. Likewise, biotypes I and II were the most prevalent among invasive strains. Biotype I, serotype b, for instance, is often associated with severe

Table 1. Frequency distribution of source of isolates and serotypes of 229 Haemophilus influenzae strains isolated from 1990 to 2003.

\begin{tabular}{|c|c|c|c|c|c|c|c|c|c|c|}
\hline \multirow[t]{2}{*}{ Year of isolation } & \multicolumn{3}{|c|}{ Source } & \multicolumn{7}{|c|}{ Serotypes } \\
\hline & CFS & B & BS & a & $b$ & c & $d$ & e & $f$ & NT \\
\hline 1990-1999 & 167 & 0 & 0 & 2 & 165 & 0 & 0 & 0 & 0 & 0 \\
\hline 2001 & 3 & 0 & 0 & 0 & 3 & 0 & 0 & 0 & 0 & 0 \\
\hline 2002 & 9 & 2 & 16 & 0 & 14 & 0 & 1 & 1 & 1 & 10 \\
\hline 2003 & 10 & 1 & 21 & 3 & 16 & 3 & 2 & 0 & 0 & 8 \\
\hline Total & 189 & 3 & 37 & 5 & 198 & 3 & 3 & 1 & 1 & 18 \\
\hline
\end{tabular}

CFS = cerebrospinal fluid; $\mathrm{B}=$ blood; $\mathrm{BS}=$ bronchial secretion; NT = non-typeable . 
Table 2. Frequency distribution of biotypes of 165 Haemophilus influenzae strains isolated from 1990 to 2003.

\begin{tabular}{lrrrrrrrr}
\hline Year of isolation & \multicolumn{7}{c}{ Biotypes } \\
\cline { 2 - 9 } & \multicolumn{1}{c}{ I } & II & III & IV & V & VI & VII & VIII \\
\hline $1990-1999$ & 47 & 26 & 3 & 5 & 4 & 9 & 3 & 6 \\
2001 & 1 & 1 & 0 & 1 & 0 & 0 & 0 & 0 \\
2002 & 14 & 7 & 0 & 0 & 3 & 2 & 1 & 0 \\
2003 & 10 & 9 & 5 & 3 & 0 & 0 & 1 & 4 \\
Total & 72 & 43 & 8 & 9 & 7 & 11 & 5 & 10 \\
& $43.6 \%$ & $26.1 \%$ & $4.9 \%$ & $5.5 \%$ & $4.2 \%$ & $6.7 \%$ & $3.0 \%$ & $6.0 \%$ \\
\hline
\end{tabular}

meningitis in children $(4,16)$. Worthy of note is the finding that in 1990-1999 the higher prevalence was of biotypes I and II. After the introduction of the conjugate vaccine (1999), a higher variability of biotypes was observed among Hi isolates, including biotypes III, IV and VIII (Table 2). The higher variability of biotypes and the various clinical manifestations caused by non-b Hi and non-typeable strains may reflect an apparent relationship between biotype and site of infection which should be evaluated by further epidemiological studies (17).

These considerations emphasize the need for close clinical, epidemiological, and laboratory surveillance using sensitive methods for determining the genetic patterns of circu- lating strains and their temporal evolution. The continued monitoring of all invasive infections caused by Hi in Brazil is imperative in order to provide accurate epidemiological data that will permit more effective measures in immunization campaigns. It is also important to reevaluate the current immunization schedules or even to identify modifications in the conjugate Hib vaccine currently in use in order to also protect against invasive disease caused by $H$. influenzae strains of any serotype, as well as non-typeable Hi strains.

\section{Acknowledgments}

We are grateful to Dr. C. Frasch, Division of Bacterial Products, Center for Biologic Evaluation and Research (CBER), Food and Drug Administration/FDA, Bethesda, MD, USA, for scientific suggestions, a critical review and helpful comments during the preparation of the manuscript. We also thank Nadjla F. de Souza and Rita Bertoncini, Public Health Laboratory/LACEN, Pernambuco and Santa Catarina States, and Cléia M.M. Cunha, Instituto Fernandes Figueira, Fundação Oswaldo Cruz, and the State Secretary of Health, Rio de Janeiro, for providing $H$. influenzae strains.

\section{References}

1. FUNASA - Boletim Eletrônico Epidemiológico. http://www.funasa. gov.br. Accessed June 12, 2003.

2. Heath PT, Booy R, Azzopardi HJ, Slack MPE, Fogarty J, Moloney AC, Ramsay ME \& Moxon ER (2001). Non-type b Haemophilus influenzae disease: clinical and epidemiologic characteristics in the Haemophilus influenzae type b vaccine era. Pediatrics Infectious Disease Journal, 20: 300-305.

3. Mühlemann K, Balz M, Aebi S \& Schopfer K (1996). Molecular characteristics of Haemophilus influenzae causing invasive disease during the period of vaccination in Switzerland: Analysis of strains isolated between 1986 and 1993. Journal of Clinical Microbiology, 34: 560-563.

4. Campos J (1999). Haemophilus. In: Murray PR, Barron EJ, Pfaller MA, Tenover FC \& Yolken RH (Editors), Manual of Clinical Microbiology. American Society for Microbiology, Washington, DC, USA.

5. Gomez-de-Leon P, Santos JI, Caballero J, Gómez D, Espinosa LE,
Moreno I, Piñero D \& Cravioto A (2000). Genomic variability of Haemophilus influenzae isolated from Mexican children determined by using enterobacterial repetitive intergenic consensus sequences and PCR. Journal of Clinical Microbiology, 38: 2504-2511.

6. Ministério da Saúde (1998). Meningites em geral. In: Guia de Vigilância Epidemiológica. Fundação Nacional de Saúde, Brasília, DF, Brazil.

7. Ribeiro GS, Reis JN, Cordeiro SM et al. (2003). Prevention of Haemophilus influenzae type b (Hib) meningitis and emergence of serotype replacement with type a strains after introduction of immunization in Brazil. Journal of Infectious Diseases, 187: 109-116.

8. Simões LLP, Andrade ALSS, Laval CA, Oliveira RM, Silva AS, Martelli CM, Alves SLA, Almeida RM \& Andrade JG (2004). Impact of Haemophilus influenzae b $(\mathrm{Hib})$ vaccination on meningitis in central Brazil. Revista de Saúde Pública, 38: 664-670.

9. LaClaire LL, Tondella MLC, Beall DS, Noble CA, Raghunathan PP, 
Rosenstein NE \& Popovic T (2003). Identification of Haemophilus influenzae serotypes by standard slide agglutination serotyping and PCR-based capsule typing. Journal of Clinical Microbiology, 41: 393396.

10. Hargreaves RM, Slack MPE, Howard AJ, Anderson E \& Ramsay ME (1996). Changing patterns of invasive Haemophilus influenzae disease in England and Wales after introduction of the Hib vaccination programme. British Medical Journal, 312: 160-161.

11. Steinhoff M \& Goldblatt D (2003). Conjugate Hib vaccines. Lancet, 361: 360-361.

12. Perdue DG, Bulkow LR, Gellin BG, Davidson M, Petersen KM, Singleton RJ \& Parkinson AJ (2000). Invasive Haemophilus influenzae disease in Alaskan residents aged 10 years and older before and after infant vaccination programs. Journal of the American Medical Association, 283: 3089-3094.

13. Urwin G, Krohn JA, Deaver-Robinson K, Wenger JD \& Farley MM (1996). Invasive disease due to Haemophilus influenzae serotype f: clinical and epidemiologic characteristics in the $H$. influenzae sero- type b vaccine era. The Haemophilus influenzae Study Group. Clinical Infectious Diseases, 22: 1069-1076.

14. McVernon J, Morgan P, Mallaghan C, Biswas T, Natarajan M, Griffiths D, Slack M \& Moxon R (2004). Outbreak of Haemophilus influenzae type b disease among fully vaccinated children in a daycare center. Pediatric Infectious Disease Journal, 23: 38-41.

15. Anonymous (2002). Progress toward elimination of Haemophilus influenzae type b invasive disease among infants and children United States, 1998-2000. Morbidity and Mortality Weekly Report, 51: 234-237.

16. Harper JJ \& Tilse MH (1991). Biotypes of Haemophilus influenzae that are associated with noninvasive infections. Journal of Clinical Microbiology, 29: 2539-2542.

17. Moustaoui N, Aitmhand R, Elmdaghri N \& Benbachir M (2000). Serotypes, biotypes and antimicrobial susceptibilities of Haemophilus influenzae isolated from invasive disease in children in Casablanca. Clinical Microbiology and Infection, 6: 48-49. 